\title{
Improving initial infectivity of the Turnip mosaic virus (TuMV) infectious clone by an mini binary vector via agro-infiltration
}

\author{
Yen-Yu Lin ${ }^{1,4+}$, Meng-Mei Fang ${ }^{1 \dagger}$, Pin-Chun Lin ${ }^{1}$, Ming-Tzu Chiu ${ }^{1,4}$, Li-Yu Liư ${ }^{5}$ Chan-Pin Lin ${ }^{4}$ and Shih-Shun Lin ${ }^{1,2,3^{*}}$
}

\begin{abstract}
Background: The in vivo infectious clone of Turnip mosaic virus (TuMV), p35S-TuMV, was used on plant pathology research for many years. To activate p35S-TuMV, the plasmid was mechanically introduced to the local lesion host Chenopodium quinoa. However, low infectivity occurred when the TuMV from C. quinoa was transferred to the systemic host Nicotiana benthamiana.

Results: To increase the efficiency of initial infectivity on N. benthamiana, the expression of the TuMV infectious clone by a binary vector that directly activates viral RNA through agro-infiltration is considered to be a good alternative. The size of the binary vector by agro-infiltration is usually large and its backbone has numerous restriction sites that increase difficulty for construction. In this study, we attempted to construct a mini binary vector (pBD003) with less restriction sites. The full-length cDNA of TuMV genome, with or without green fluorescence protein, was inserted in pBD003 to generate PBD-TuMV constructs, which were then individually introduced to N. benthamiana plants by agro-infiltration. Symptom development and ELISA positivity with TuMV antiserum indicated that the pBD-TuMV constructs are infectious. Moreover, the initial infectivity of a mild strain TuMV-GK, which contains an $\mathrm{R}_{182} \mathrm{~K}$ mutation on HC-Pro, constructed in the pBD003 vector was significantly increased by agro-infiltration.
\end{abstract}

Conclusion: Thus, we concluded that the newly constructed mini binary vector provides a more feasible tool for TuMV researches in areas, such as creating a mild strain for cross-protection, or a viral vector for foreign gene expression. In addition, the multiple cloning sites will be further cloned in pBD003 for convenience in constructing other viral infectious clones.

Keywords: Turnip mosaic virus; Infectious clone; Initial infectivity; Mini binary vector

\section{Background}

The infectious clone of the RNA virus is a plasmid containing the full-length cDNA form of the viral genome. Under a suitable promoter, the DNA form of a viral genome can be transcribed into an initial infectious viral RNA by in vitro or in vivo methods to enable research into molecular virology. The viral infectious clone was developed in the late 1970s, and benefited by the recombinant DNA and reverse-transcription techniques

\footnotetext{
*Correspondence: linss01@ntu.edu.tw

${ }^{\dagger}$ Equal contributors

'Institute of Biotechnology, College of Bioresources and Agriculture, National Taiwan University, 81, Chang-Xing ST., Taipei 106, Taiwan

${ }^{2}$ Genome and Systems Biology Degree Program, National Taiwan University,

1, Sec. 4, Roosevelt Rd., Taipei 106, Taiwan

Full list of author information is available at the end of the article
}

(Boyer and Haenni, 1994). Currently, in an infectious clone, it is possible to introduce a mutation on the viral genome at a particular position, or to process recombination between two strains of a particular virus. The infectious clone has become an indispensable tool in the research of plant and animal viruses (Kobayashi et al., 2007; Schnell et al., 1994).

Initial infectivity refers to the triggering of transcription of the DNA form of a viral genome to generate infectious viral RNA, which begins to establish the life phase of a virus. Thereafter, the viral RNA can self-replicate and has the ability to subsequent infect the host plant. Based on differences in initial processing, infectious clones are classified as either in vitro or in vivo. The in vitro infectious clones require phage promoters, such as $T 7, T 3$, or $S p 6$ promoter to generate viral RNA by in vitro transcription. 
In contrast, the CaMV $35 S$ promoter is usually used with the in vivo infectious clone and the promoter is recognized by host RNA polymerase II (pol II) in the plant nucleus to transcribe viral RNA in vivo. (Angenent et al., 1989; Heaton et al., 1989; Janda et al., 1987; Lin et al., 2002; Melton et al., 1984). Once the viral RNA is transcribed from DNA, the virus life cycle is initiated, and consequent infectivity is established.

The initial infectivity rate may be affected by RNase contamination, inoculation method, host characteristics, and viral activity. The RNase contamination specifically indicated that the operation with the in vitro transcript that was exposed to an RNase-rich environment might damage the viral RNA during the inoculation. In a previous study, plants of Chenopodium quinoa were inoculated with a DNA form of an in vivo infectious clone of Zucchini yellow mosaic virus (ZYMV) by mechanical inoculation for local lesion formation, and the generated virus was then transferred from a $C$. quinoa plant to a zucchini squash plant (Lin et al., 2002). It has been reported that the host characteristics of squash cannot cause initial infectivity by direct mechanical inoculation with the in vivo infectious clone of ZYMV (Lin et al., 2002). The C. quinoa plants can be directly inoculated with the DNA of an in vivo infectious clone, with high efficiency of the infectivity and without the risk of RNase contamination. This process is easy and less costly than in vitro transcription. However, the secondary inoculation for the transfer of a virus from C. quinoa to a systemic host plant is somehow difficult especially for a viral mutant, which does not form distinct local lesions. The Turnip mosaic virus (TuMV) has low infectivity on Nicotiana benthamiana plants when the inoculum is prepared from infected $C$. quinoa tissue (unpublished data). It appears that the TuMV activity was affected by an unknown-mechanism when the virus existed in C. quinoa. In addition, the inefficiency of the initial infectivity of TuMV (less than a 10\% success rate) in $N$. benthamiana plants caused by particle bombardment with an in vivo TuMV infectious clone was also a problem (unpublished data). These difficulties generate a limitation for studying the functions of TuMV mutants, in which weaker infectivity may be incurred by mutations. In addition, similar to ZYMV, the DNA of the in vivo infectious clone of TuMV also does not efficiently cause initial infectivity on the systemic host $N$. benthamiana by mechanical inoculations.

To solve this problem, we attempted to transfer the in vivo infectious clone into the nucleus of a host plant by agro-infiltration, thus bypassing the need for $C$. quinoa inoculation. The binary vector was designed for Agrobacterium tumefaciens to transfer the T-DNA into a plant cell nucleus (Joh and VanderGheynst, 2006). To date several in vivo infectious clones have been designed to deliver by the binary vector through agro-infiltration, such as Citrus yellow mosaic virus (CYMV), Tobacco rattle virus (TRV), and Begomovirus, to increase the initial infectivity rate in $N$. benthamiana (Cui et al., 2005; Huang and Hartung, 2001; Liu et al., 2002). Agro-infiltration provides a good strategy to overcome the lower rate of initial infectivity. However, the size of the binary vector is usually large (>10 kb) and difficulties may be encountered in ligation with large potyviral genome inserts, which are usually approximately $10 \mathrm{~kb}$ in length. In addition, numerous commonly used restriction enzyme sites exist on the backbone of the binary vector, limiting the construction strategy with regard to the use of restriction sites.

In this study, we constructed a mini binary vector $(4 \mathrm{~kb})$, pBD003, in which most of restriction enzyme sites on the plasmid backbone were removed. The full length of TuMV cDNA was constructed in pBD003, downstream from the CaMV $35 S$ promoter, with suitable restriction enzyme digestion and ligation to generate an in vivo pBD-TuMV infectious clone. We further generated pBDTuMV-GFP, which carries a green fluorescent protein $(G F P)$ gene for tracking the virus in host. In addition, a TuMV mild strain, TuMV-GK, which has $\mathrm{R}_{182} \mathrm{~K}$ mutation on HC-Pro, was also constructed in pBD003 vector in this study. The biological activities of the pBD-TuMV series were analyzed on the local lesion host $C$. quinoa by mechanical inoculation and on the systemic host $N$. benthamiana by agro-infiltration. The initial infectivity of these pBD-TuMV series was detected and confirmed by indirect enzyme-linked immunosorbent assay (ELISA), which verified the infectivity of these infectious clones. Thus, these pBD-TuMV series of infectious clones provide a powerful tool for further research into topics, such as creating a mild strain for cross-protection. Multiple cloning sites (MCS) will be introduced in pBD003 in the future, and will be suitable for other viral infectious clone construction.

\section{Methods}

\section{Plant material and growth condition}

Plant-related experiments, such as agro-infiltration and viral inoculation were performed in a greenhouse ( $16 \mathrm{~h}$ light $/ 8 \mathrm{~h}$ darkness, $20^{\circ} \mathrm{C}$ to $25^{\circ} \mathrm{C}$ ). The seeds of C. quinoa and $N$. benthamiana were sown in peat soil. After germination, seedlings were transferred to soil and fertilized with Peters 20-20-20 once a week.

\section{Construction of a mini binary vector}

The strategy for constructing a mini binary vector with the $35 S$ promoter and nos terminator is summarized in Figure 1 . The $p S a$ origin was amplified by polymerase chain reaction (PCR) with the primer pair FP-pSa-SpeI (5' - TC TTATCACTAGTAAGCCCGAGAGGTTGCCGCC -3') and RP-pSa-NheI (5' - GGAGGGTAGGCTAGCGTTATC CACGTGAAACCGC -3'), which contain SpeI and NheI 


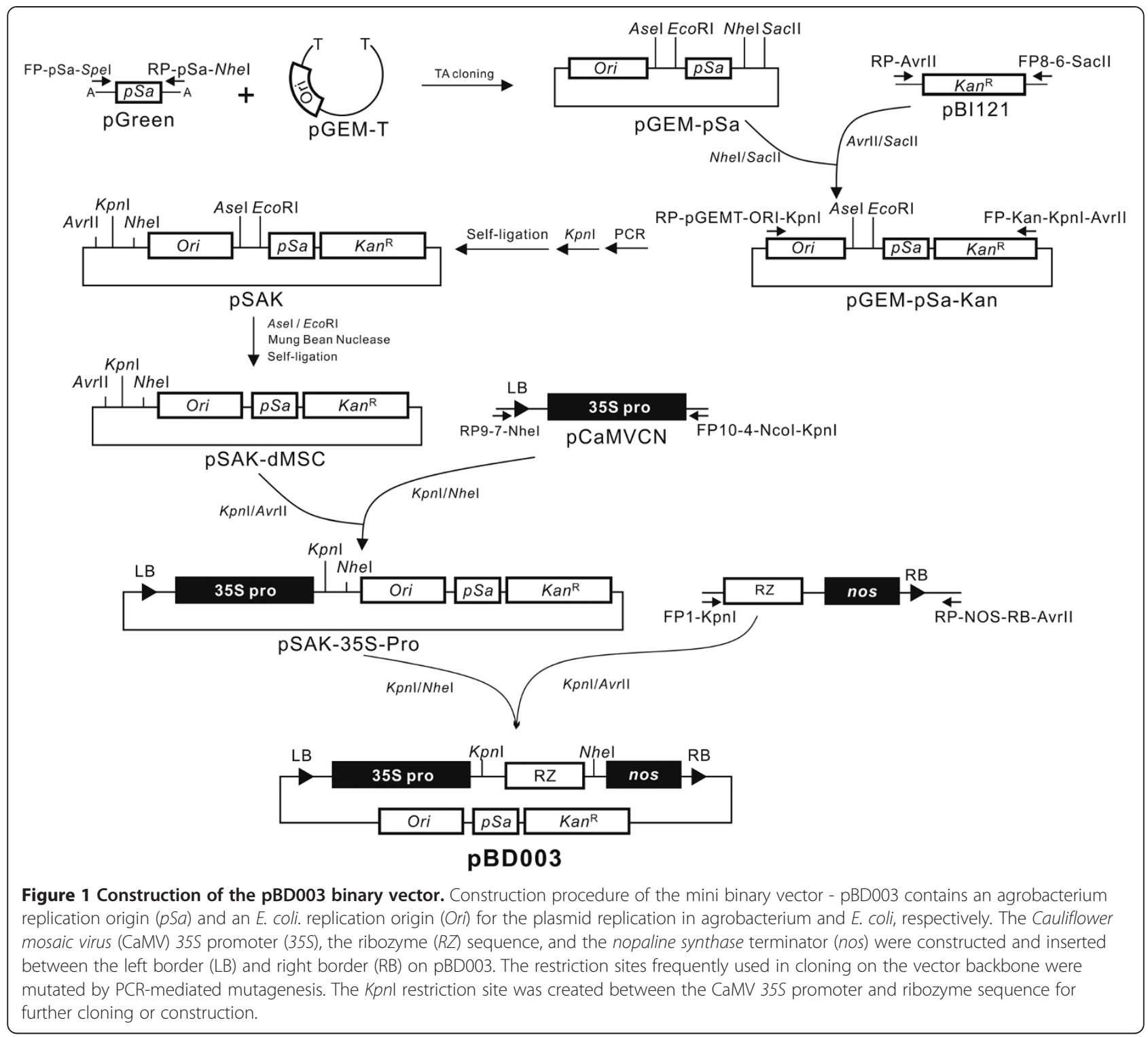

(underlined), respectively, from pGreen (Hellens et al., 2000) and was cloned into the pGEM-T vector (Promga) to generate pGEM-pSa. Next, the kanamycin resistance gene $\left(K^{2} n^{\mathrm{R}}\right)$ was amplified from pBI121 binary vector with the primer pair FP-8-6-SacII (5'- GCCTGTGATCAT CCGCGGTTTCAAAATCGGCTCCG -3') and RP-AvrII (5'- GGTTTTTTTGTTTGCAAGCCTAGGCAGATTAC GCGC - $3^{\prime}$ ), which contains SacII and AvrII (underlined), respectively. The PCR fragment of $\mathrm{Kan}^{\mathrm{R}}$ gene was digested with AvrII/SacII and ligated to NheI/SacII-digested pGEM-pSa vector to generate pGEM-pSa-Kan. To remove the unnecessary DNA backbone, the primer pair FPKan-KpnI-AvrII (5' - GCTCCCGGTACCGCCAGGCG GCCTAGGTTTCAAAATCGG -3') containing KpnI and AvrII (underlined), and RP-pGEMT-ORI-KpnI (5' - GCCT CACTGATTAAGCATTGGTACCTGTCAGACC -3') containing KpnI, (underlined) was used to amplify the 2,916 bp fragment of pGEM-pSa-Kan that included the Ori-pSa-Kan ${ }^{R}$ sequence by PCR. The PCR product was digested with $K p n \mathrm{I}$ and self-ligated to generate pSAK. Thereafter, the pSAK plasmid was digested with AseI/ EocRI to remove the restriction enzyme sites and the digested product was treated with Mung Bean nuclease to produce blunt ends and then self-ligated to generate pSAKdMSC. The left border (LB) and CaMV $35 S$ promoter sequence were amplified from pCaMVCN (Pharmacia) with the primers FP10-4-NcoI-KpnI (5'- CCGGCCG CCATGGGGTACCCCTCTCCAAATGAAAT -3') and RP9-7-NheI (5' - GCGATGATCACAGGCTAGCAACGC TCTGTCATCG -3'), which contain KpnI/NcoI, and NheI (underlined), respectively. The PCR product was then digested with KpnI/NheI and ligated with KpnI/ 
AvrII-digested pSAK-dMSC vector to generate pSAK35S-Pro. Finally, the DNA region including the ribozyme, nos, and right border (RB) was amplified from pTRV2 plasmid (Liu et al., 2002) with the primers FP1-KpnI (5' - ACTTGGTACCGTCTGTACTTATATCAGTACACT GACGAG -3') and RP-NOS-RB-AvrII (5' - TTGGCACC TAGGTACAAATGGACGAACGGATAAAC -3'), which contain $K p n I$, and AvrII (underlined), respectively. The PCR product was digested with $K p n \mathrm{I} / A v r \mathrm{II}$ and ligated with the KpnI/NheI-digested pSAK-35S-Pro vector to generate the mini binary vector pBD003 of 4,141 bp.

\section{Construction of in vivo full-length CDNA clones of TuMV for agro-infiltration}

The strategy for constructing the pBD-TuMV serial in vivo infectious clones is summarized in Figure 2. Two in vivo TuMV infectious clones, p35S-TuMV-YC5 and p35STuMV-GFP, constructed on the pCaMVCN vector were provided by Dr. Shyi-Dong Yeh (Figure 2A) (Lin et al., 2009; Niu et al., 2006). The 3 '-end fragment of the TuMV was amplified by PCR with the primers FP-TuCP9353-KpnI (5' -AACCGGTACCGACCATACATGCCACGATATGGT CTTC-3') and RP-POLYA-AvrII (5'-AGGTCGACGCG GCCGCCTAGGTTTTTTTTTTTTTTT-3'), which contain $K p n \mathrm{I}$, and $A v r I I$ (underlined), respectively. The PCR fragment was digested with KpnI/AvrII and then ligated to the KpnI/NheI-digested pBD003 vector to generate pBD-TuCP. Next, the EcoRV/KpnI-digested fragment from p35S-TuMV-YC5 was ligated into the EcoRV/KpnI-digested $\mathrm{pBD}-\mathrm{TuCP}$ vector to generate pBD-TuMV5'3'-YC5. The primers FP-TuMV7804-KpnI (5' - GGAAAGGTACCCGTGGATGATTTCAACAAC -3') containing KpnI (underlined), and MTuCP8854 (5'- GC CTCTCTCGTTCCTTTTCT -3') were used to amplify the region between the $N I b$ and $C P$ genes from p35STuMV-YC5 and p35S-TuMV-GFP, respectively. The KpnI/MluI-digested fragment from p35S-TuMV-YC5 was ligated with the same restriction enzyme-digested pBD-TuMV-5'3'-YC5, to generate pBD-TuMV5' 3'X-YC5. In addition, the KpnI/MluI-digested fragment from p35S-TuMV-GFP was ligated with KpnI/MluI-digested pBD-TuMV-5'3'-YC5 to generate pBD-TuMV-5'3'XGFP. Finally, the fragment that was digested by KpnI/ XhoI from p35S-TuMV-YC5 was ligated with the same enzyme-digested pBD-TuMV-5' $3^{\prime} \mathrm{X}-\mathrm{YC} 5$ and $\mathrm{pBD}-\mathrm{TuMV}$ $5^{\prime} 3^{\prime} X-G F P$ to generate pBD-TuMV-YC5 and pBD-TuMVGFP, respectively (Figure $2 \mathrm{~B}$ ).

For the mild strain of TuMV construction, the $\mathrm{R}_{182} \mathrm{~K}$ mutation was introduced on the HC-Pro gene by PCR mutagenesis on p35S-TuMV-YC5, following the method demonstrated by Lin et al. (2009). The HC-Pro ${ }^{\mathrm{R} 182 \mathrm{~K}}$ sequence was digested with $M f e \mathrm{I} / K p n \mathrm{I}$ and ligated with the same enzyme-digested pBD-TuMV-GFP to generate pBD-TuMV-GK (Figure 2C).

\section{Infectivity assay of in vivo full-length cDNA clones}

Local lesion host $C$. quinoa and systemic host $N$. benthamiana were used for the in vivo infectious clone infectivity assay. An aliquot of $10 \mu \mathrm{l}(1 \mu \mathrm{g} / \mu \mathrm{l})$ of individual constructs were mechanically introduced to leaves of $C$. quinoa with carborundum-dust, and the development of local lesions was recorded at 7 days post-inoculation (dpi). The mock (control) was mechanically inoculated with $10 \mu \mathrm{l}$ $\mathrm{ddH}_{2} \mathrm{O}$. For the agrobacterium mediated transformation activity assay, the Agrobacterium tumefaciens strain C58C1, which carries pBD-TuMV serial in vivo infectious clones, was incubated in an LB medium supplemented with kanamycin $(100 \mu \mathrm{g} / \mu \mathrm{l})$ at $28^{\circ} \mathrm{C}$ for $16 \mathrm{~h}$. Thereafter, agroinfiltration was applied following the standard protocol (Llave et al., 2000). The symptoms and GFP fluorescence were observed after 6 days of infiltration.

\section{Purification of recombinant TuMV CP and production of antisera}

The TuMV coat protein $(C P)$ gene was amplified from the p35S-TuMV-YC5 infectious clone with primers PTu-CPNdeI (5' - GTGTTTATCATATGGCAGGTGAGACG -3') and MTu-CP-XhoI (5' - CAACTTCACTCGAGCTATAA CCCCTTAACGC -3'), which contain NdeI, and XhoI (underlined), respectively. The PCR fragment was digested with $\mathrm{NdeI} / \mathrm{XhoI}$ and ligated with the same restriction enzyme-digested pET-28b to generate pET-TuCP. The pET-TuCP was transferred into E. coli BL21 (DE3), which was treated with $0.5 \mathrm{M}$ IPTG in LB medium for recombinant his-TuCP production. The recombinant his-TuCP was purified with Ni-NTA column by fast protein liquid chromatography (FPLC) (AKTApurifier, GE Healthcare) and used for antisera production that were produced from New Zealand white rabbits, as described by Lin et al. (2002). The titer of TuMV CP antisera was analyzed by Enzyme-linked immunosorbent assay (ELISA) and western blotting.

\section{Enzyme-linked immunosorbent assay (ELISA)}

For verification of virus infection, each sample represented 3 inoculated leaves and 3 systemic leaves collected from each of the 3 repeated plants, with 3 leaf-discs $(0.6 \mathrm{~cm}$ in diameter) punched from each leaf. These collected samples were assayed by indirect ELISA using the polyclonal antiserum to the TuMV CP. Goat anti-rabbit immunoglobulin $\mathrm{G}$ conjugated with alkaline phosphatase (Amersham Biosciences) was used as the secondary antibody, and $p$-nitrophenyl phosphate (Sigma) was used as the substrate for color development. Results were recorded by measuring absorbance at $405 \mathrm{~nm}$ (which were stained for 5 min after the addition of the substrate) using an ELISA reader (Perkin Elmer).

The analysis of variance (ANOVA) was performed at first to test the levels of absorbance under different 


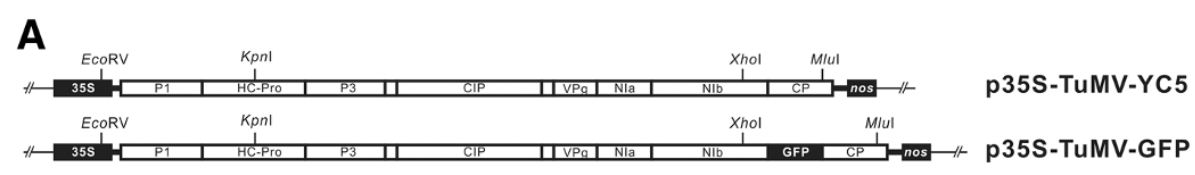

\section{B}
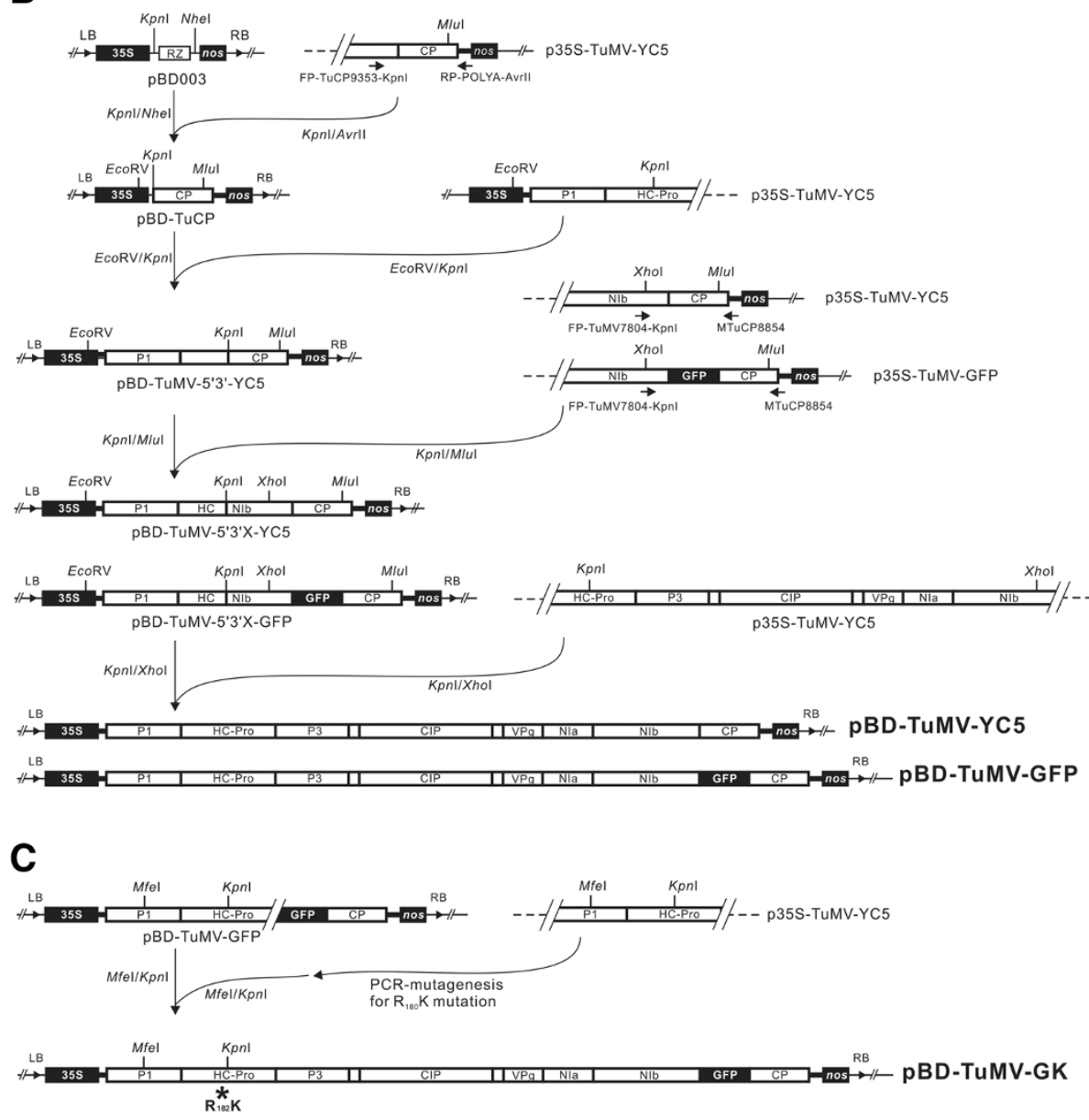

Figure 2 Schematic representation of constructing pBD-TuMV infectious clones. (A) Two TuMV in vivo infectious clones, p35S-TuMV-YC5 and p35S-TuMV-GFP (Lin et al., 2009; Niu et al., 2006), constructed on the pCaMVCN vector (Pharmacia) were designed for mechanical inoculation on C. quinoa plants. The two clones were used in the current study to construct a series of infectious constructs using a binary vector for agroinfiltration. (B) The full-length cDNA constructs of TuMV were constructed in the pBD003 binary vector (pBD-TuMV-YC5 and pBD-TuMV-GFP) for agrobacterium infiltration on $\mathrm{N}$. benthamiana plants. The construction steps for cloning the TuMV full-length cDNA into the pBD003 binary vector are shown. The full-length TuMV cDNAs released from the p35S-TuMV series were constructed downstream of the CaMV 35S promoter (35S) by insertions in between suitable restriction enzyme sites of pBD003. The final constructs were designated pBD-TuMV-YC5 and pBD-TuMV-GFP, contained a complete CDNA copy of wild type TUMV and TuMV carrying GFP, respectively. The coding regions of the cDNA are indicated by an open box. The 5' and 3' non-coding regions of the CDNA are indicated by heavy lines. (C) R ${ }_{182} \mathrm{~K}$ mutation on HC-Pro of the TuMV was conducted by PCR-mediated mutagenesis. The mutated fragment was digested with Mfel/Kpnl and replaced the corresponding region of pBD-TuMV-GFP to generate PBD-TuMV-GK. An asterisk indicated the $\mathrm{R}_{182} \mathrm{~K}$ mutation position.

conditions. It was followed by the pairwise comparison between two conditions of interest using Tukey's honest significant difference (HSD) test.

\section{Western blot analysis}

The systemic leaves from inoculated plants were homogenized in 20 volumes (wt/vol) of denaturing buffer $(50 \mathrm{mM}$ Tris-HCl, pH 6.8, 4\% SDS, 2\% 2-mercaptoethanol, 10\% glycerol, and $0.001 \%$ bromophenol blue). After incubation at $100^{\circ} \mathrm{C}$ for $5 \mathrm{~min}$, extracts were clarified by centrifugation at $8,000 \mathrm{~g}$ for $3 \mathrm{~min}$. Total proteins were separated by SDS gel electrophoresis, and western blots were analyzed using the antiserum to TuMV CP. Gels were stained with Coomassie brilliant blue R250, and levels of the large subunit of RUBISCO (molecular mass, $55 \mathrm{kDa}$ ) were used as loading controls. 


\section{Results}

\section{Construction of a mini binary vector - pBD003}

The $4 \mathrm{~kb}$ mini binary vector, pBD003, contains LB and $\mathrm{RB}$ sequences. Between the $\mathrm{LB}$ and the RB sequences, the construct contains a CaMV $35 S$ promoter, a kanamycin resistance gene $\left(\mathrm{Kan}^{R}\right), p S a$ origin, E. coli replication origin (Ori), nos terminator, and ribozyme site $(R Z)$ (Figure 1). The role of nos terminator is to help generate a polyA tail for the polyA-type viruses, such as potyvirus, whereas the ribozyme site is designed for the non-polyA viruses. In addition, commonly used restriction enzyme sites were removed from the pBD003 backbone, which reduces the problems of introducing inserts for cloning purposes (Figure 1).

\section{Construction of full-length CDNA clones with 355 promoter in the mini binary vector}

The construction of three pBD-TuMV clones was based on two plasmids, p35S-TuMV-YC5, and p35S-TuMVGFP. The p35S-TuMV series all contain full-length TuMV cDNA between the CaMV $35 S$ promoter and nos terminator (Figure 2A) in which the ribozyme sequence was removed during this construction. The p35S-TuMVGFP contains the GFP gene between NIb and CP genes of the TuMV (Figure 2A).

The pBD-TuMV series contained the full-length cDNA sequence of TuMV-YC5 downstream from the $35 \mathrm{~S}$ promoter and located between LB and RB based on the binary vector $\mathrm{pBD} 003$. The scheme for the construction of TuMV full-length clones in the binary vector pBD003 from the p35S-TuMV series is shown in Figure 2B. Moreover, the $\mathrm{R}_{182} \mathrm{~K}$ mutant of HC-Pro was created on pBD-TuMVGFP clone by PCR-mediated mutagenesis to generate $\mathrm{pBD}-$ TuMV-GK (Figure 2C). The pBD-TuMV-GK was used for further investigation in this study.

\section{Initial infectivity assay of in vivo full-length CDNA clones on C. quinoa plant}

To test the infectivity of the pBD-TuMV serial clones, $C$. quinoa plants were mechanically inoculated with individual pBD-TuMV plasmids. Local lesions appeared after $7 \mathrm{dpi}$, whereas the mock did not show any local lesions (Figure 3A). Moreover, green fluorescence appeared on the leaves inoculated with pBD-TuMV-GFP and pBD-TuMVGK, whereas no green fluorescence appeared on the pBDTuMV-YC5 or mock-inoculated leaves (Figure 3A, lower panel). In addition, the virus culture of TuMV YC5 was also mechanically inoculated on $C$. quinoa plant as a positive control (Figure 3A); the lesion morphology and developing time was identical to the inoculation of the pBD-TuMVYC5 plasmid. These results indicated that the $3 \mathrm{pBD}-$ TuMV plasmids were infectious, and that pBD-TuMV-GFP and pBD-TuMV-GK expressed the GFP gene.
The TuMV mild strain (TuMV-GK) with $\mathrm{R}_{182} \mathrm{~K}$ mutation on the HC-Pro gene showed mild symptoms on $N$. benthamiana plants (Figure 3B). Chlorotic spots appeared on TuMV-GK-infected C. quinoa without necrosis (Figure 3A). In addition, the TuMV-GK infected tissue showed weaker green fluorescence in both plants compared with those produced by WT TuMV (Figure 3). Moreover, the WT TuMV produced green fluorescence spots on $C$. quinoa, but TuMV-GK produced a green fluorescence ring on C. quinoa (Figure 3A).

\section{Transformation analysis by agro-infiltration}

The biological activity of the pBD003 binary vector for the agrobacterium-mediated transformation was tested, and the infectivity of the pBD-TuMV series was evaluated on the TuMV systemic host $N$. benthamiana by agroinfiltration, whereas the virus culture of TuMV YC5 was mechanically inoculated on $N$. benthamiana plant as a positive control. The pBD-TuMV-GFP successfully infected the systemic host $N$. benthamiana by agro-infiltration, with infected plants showing mosaic, necrotic spots, leaf curling and wilting symptoms (Figure 3B, upper panel). Fluorescent microscopy revealed that pBD-TuMV-GFP agro-infiltrated plants expressed green fluorescence on their leaves, whereas pBD-TuMV-YC5, the virus culture of TuMV YC5, and healthy plants showed the original red fluorescence of chloroplasts (Figure 3B, lower panel). Moreover, pBDTuMV-GK showed mild symptoms on $N$. benthamiana plants, namely mild curling of new leaves, but these symptoms recovered after a few days (Figure 3B). The results indicated that the pBD-TuMV series are infectious by agro-infiltration.

All inoculated plants were analyzed by western blot and indirect ELISA to confirm the infection. The $N$. benthamiana plants with severe symptoms reacted strongly to TuMV CP antiserum, and the healthy control showed negative reactions (Figure 4A \& B). By western blotting analysis, a $35 \mathrm{kDa}$ protein corresponding to the $\mathrm{CP}$ of TuMV was detected in plants agro-infiltrated by the TuMV in vivo infectious clones (Figure 4A). The results also indicated that the antisera to TuMV CP produced by recombinant protein can specifically detect TuMV infection as analyzed by ELISA or western blot (Figure 4).

\section{Evaluation of the initial infectivity efficiency of the pBD-TuMV-series infectious clones}

To test whether the pBD-TuMV-series infectious clones could improve initial infection efficiency, we compared the success infection rates of the $N$. benthamiana plant by agro-infiltration versus the mechanical inoculation. Here, the mechanical inoculation was used 4 local lesions of $C$. quinoa tissue as inoculums. In addition, the severe strain (TuMV-GFP) and the mild strain (TuMV-GK) were evaluated, as shown in Table 1 . The success rate for pBD- 

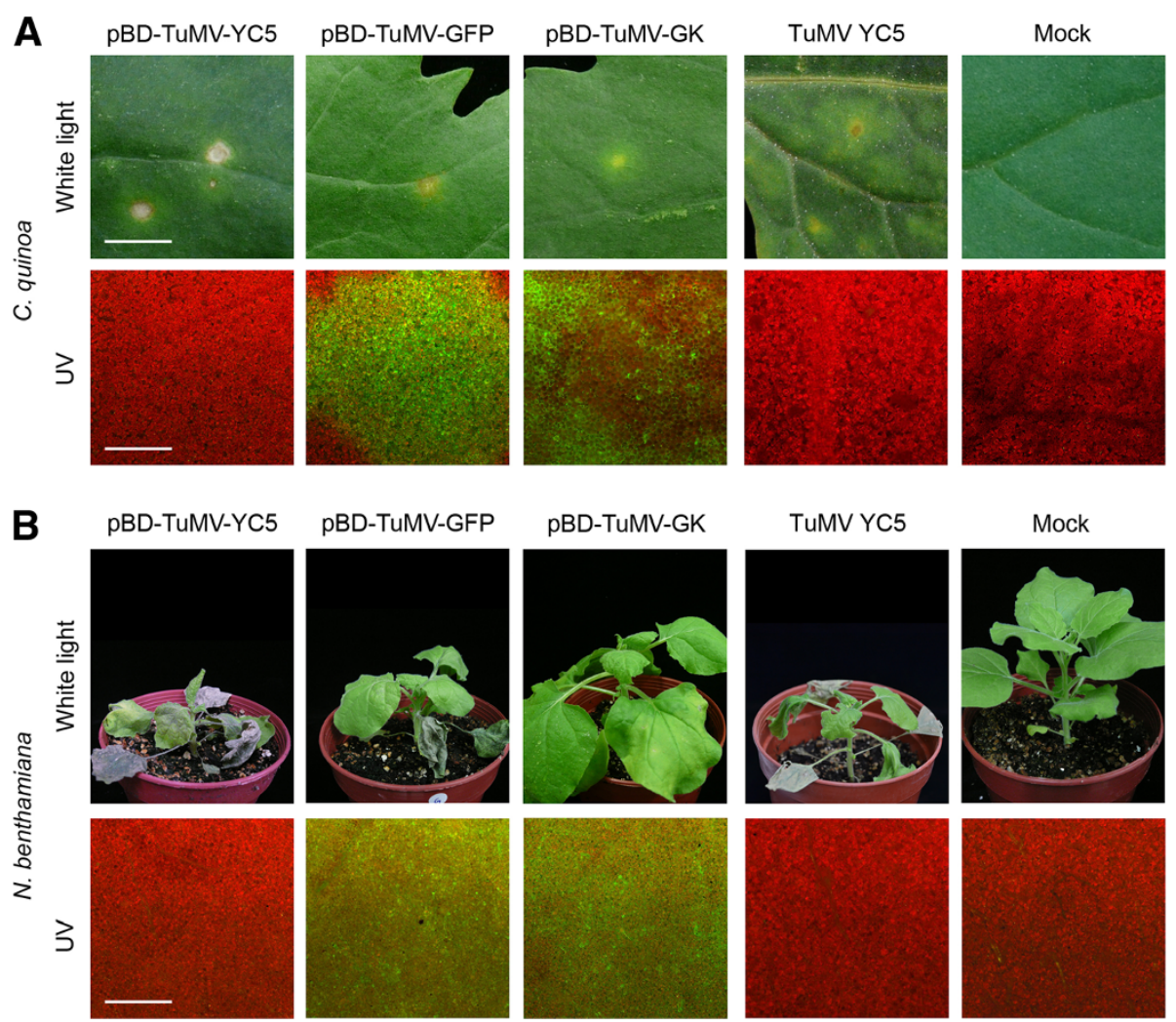

Figure 3 Infectivity assay of pBD-TuMV-YC5, pBD-TuMV-GFP and pBD-TuMV-GK. (A) The 3 constructs were directly mechanically introduced to C. quinoa plants, and local lesions appeared on the inoculated leaves at 7 dpi (upper panel). The virus culture of TuMV YC5 and Mock-inoculation were used as positive and negative controls, respectively. Bar, $0.5 \mathrm{~cm}$. The GFP fluorescence expressed by pBD-TuMV-GFP and pBD-TuMV-GK is shown in the lower panel. Bar, $25 \mu \mathrm{M}$. (B) N. benthamiana plants were infiltrated with agrobacterium individually carrying pBDTuMV-YC5, pBD-TuMV-GFP, and pBD-TuMV-GK. The virus culture of TuMV YC5 and Mock-inoculation were used as positive and negative controls, respectively. The photographs were taken at $14 \mathrm{dpi}$ (upper panel). GFP expression was detected with a fluorescent microscope (lower panel).

TuMV-GFP initial infection on N. benthamiana was $100 \%$ by agro-infiltration, whereas the success rate was only $10 \%$ by mechanical inoculation when the inoculums were taken from 4 local lesions of C. quinoa (Table 1). The results indicate a low success rate for TuMV initial infection transferred from C. quinoa plants to $N$. benthamiana; agro-infiltration eliminated this problem by directly generating the TuMV on N. benthamiana, which sped the initial infection. In addition, the data showed lower initial infectivity of TuMV-GK ( $10 \%$ success rate) using mechanical inoculation from local lesions of C. quinoa to $N$. benthamiana (Table 1). However, TuMV-GK showed a $53.3 \%$ initial infection rate on agro-infiltrated $N$. benthamiana (Table 1). This finding indicates that agroinfiltration can also eliminate the problem of low infectivity of the TuMV that has HC-Pro mutation.

\section{The pBD003 produced the bursts of transferred RNA to advance the viral RNA accumulation}

We compared the TuMV accumulated rates for mechanical inoculation with virus culture of TuMV YC5 versus agro- infiltration with pBD-TuMV-YC5 by ELISA (Figure 5). Here, the mechanical inoculation was used TuMV YC5-infected $N$. benthamiana tissue as inoculuns. The $N$. benthamiana plants infected with virus culture of TuMV YC5 by mechanical inoculation, show the leafcuring symptoms on the systemic leaves at 12 days after mechanical inoculation; the ELISA readings of TuMV CP $\left(\mathrm{OD}_{405}\right)$ showed TuMV-positive signals on the inoculated $\left(\mathrm{OD}_{405} 0.6 \pm 0.34\right)$ and systemic leaves $\left(\mathrm{OD}_{405} 1.88 \pm 0.14\right)$ (Figure 5). No significance symptoms were observed on either the inoculated leaves or systemic leaves between 0 to $6 \mathrm{dpi}$. The ELISA readings $\left(\mathrm{OD}_{405}\right)$ averaged $0.22 \pm 0.02$ at 0 to 6 days after mechanical inoculation in inoculated and systemic leaves (Figure 5). TuMV infection was not detectable during the time less than $6 \mathrm{dpi}$.

Using the agro-infiltration method with pBD-TuMVYC5, the inoculated leaves showed that the readings of $\mathrm{OD}_{405}$ were $0.4 \pm 0.07$ ( $\left.2 \mathrm{dpi}\right), 1.44 \pm 0.15$ ( $\left.4 \mathrm{dpi}\right)$, and $1.85 \pm 0.06$ ( $6 \mathrm{dpi})$ and the systemic leaves showed that the readings of $\mathrm{OD}_{405}$ were $0.41 \pm 0.04(4 \mathrm{dpi})$ and $1.3 \pm 0.16$ (6 dpi) (Figure 5). Our results indicated that the agro- 


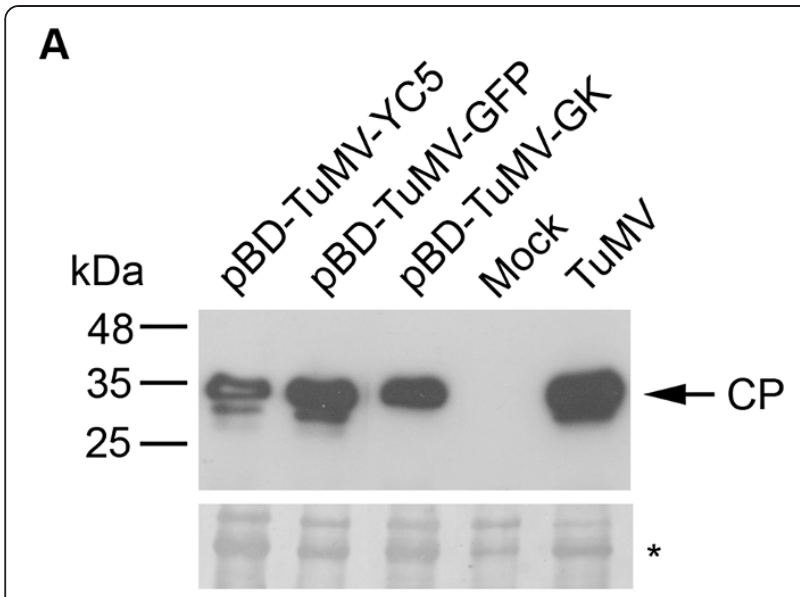

B

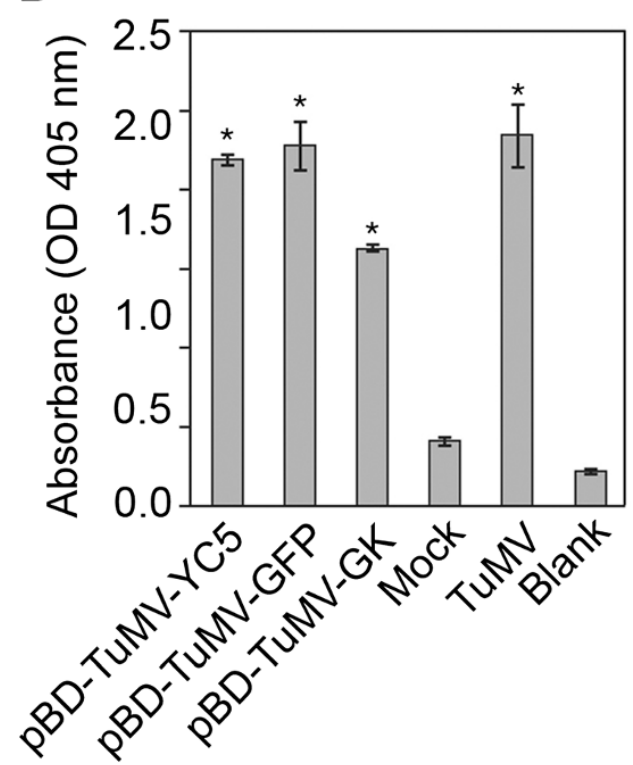

Figure 4 Validation of the infectivity of pBD-TuMV-CY5, pBD-TUMV-GFP and PBD-TuMV-GK on $N$. benthamiana plants. The agro-infiltrated $N$. benthaminana plants were analyzed with western blotting (A) or indirect ELISA (B) at 7 dpi, using the antiserum to TuMV CP. The samples of agro-infiltrated leaves are individually indicated. The sample from leaves infected by TuMV virus culture (14 dpi) that was used as positive control is indicated as TuMV. The mock was inoculation was used as a negative control. The blank indicates the wells coated with buffer for basal-level control. The TuMV CP antiserum was used at 10,000x dilution for both assays. The samples significantly different with mock ( $p$-value $<0.05$ ) were indicated as "**". Bars represent standard deviations $(n=3)$.

infiltration method results in bursts of TuMV viral RNA in inoculated leaves by the expression of pBD-TuMV-GFP resided in T-DNA, and these abounding viral RNAs enhance the TuMV systemic infection efficiency.

\section{Discussion}

A commercialized mini binary vector (pGreen) is available (Hellens et al., 2000). However, some restriction sites
Table 1 Comparison of initial infectivity for agro-infiltration versus mechanical inoculation

\begin{tabular}{lccc}
\hline Virus & $\begin{array}{c}\text { Inoculation } \\
\text { method }\end{array}$ & $\begin{array}{c}\text { Systemic } \\
\text { leaf }\end{array}$ & $\begin{array}{c}\text { Initial } \\
\text { infectivity (\%) }\end{array}$ \\
\hline TUMV-GFP & A.I. & $10 / 10$ & 100 \\
\multirow{2}{*}{ TuMV-GK } & M.I. ${ }^{\text {b }}$ & $1 / 10$ & 10 \\
& A.I. & $8 / 15$ & 53.3 \\
\hline
\end{tabular}

${ }^{\mathrm{a}}$ A.I., the inoculation method was applied by agro-infiltration with pBD-TuMVGFP or pBD-TuMV-GK on N. benthamiana plants. ${ }^{\mathrm{b}} \mathrm{M} . \mathrm{I}$, individual lesions on C. quinoa plants infected with pBD-TuMV-GFP or PBD-TuMV-GK were collected and used as inocula for mechanical inoculation. 'Number of infected plants as a percentage of the total plant population. The virus infection was analyzed by ELISA at 12 dpi.

existing on the pGreen backbone might interfere with further cloning procedures of the TuMV infectious clone. Therefore, we designed a mini binary vector that is specifically suitable for prompt construction of TuMV infectious clones. In this study, a mini binary vector (pBD003) for agrobacterium-mediated transient expression was created. The vector was then applied to deliver virus infectious clones to the nuclei of plant cells. The vector was 4,141 bp in size and unnecessary restriction sites were removed from the plasmid backbone. We used the strategy of compatible cohesive end of restriction enzymes, such as AvrII ligated with NheI, to link various comportments to avoid restriction sites remaining on the vector after ligation. The advantage of a plasmid with fewer restriction sites on the vector backbone can increase the usage of restriction sites. Moreover, having a miniature-sized vector can increase the rate of successful construction. This is especially useful when handling the large fragments for recombination analyses, genomic mutation, or inserting a foreign openreading frame into the infectious clone. The pBD003 contains a ribozyme to cleave the nascent RNA at the 3 '-end for generation of infectious clones for non-poly A tail type of viruses. In addition, pBD003 also has a nos terminator, which provides a polyadenylation signal to generate a poly A tail on viral RNA. In our case of TuMV, the ribozyme sequence has been removed in the plasmid of pBD-TuMV series.

In pBD003, the KpnI and NheI sites on the vector can be used for other viral infectious clone constructions. In the future, the more restriction enzyme sites will be introduced to the pBD003 for cloning convenience. However, these restriction sites immediately after the $35 \mathrm{~S}$ promoter must be removed by site-directed mutagenesis because the non-viral sequence between the $35 \mathrm{~S}$ promoter and $5^{\prime}$-end in viral RNA affects the initial infectivity (Lin et al., 2002; Maiss et al., 1992; Shi et al., 1997).

The plasmids of serial pBD-TuMV infectious clones can be applied directly on C. quinoa by mechanical inoculation to induce local lesions, indicating that these pBD-TuMV constructs possess viral infectivity. Moreover, the pBD- 


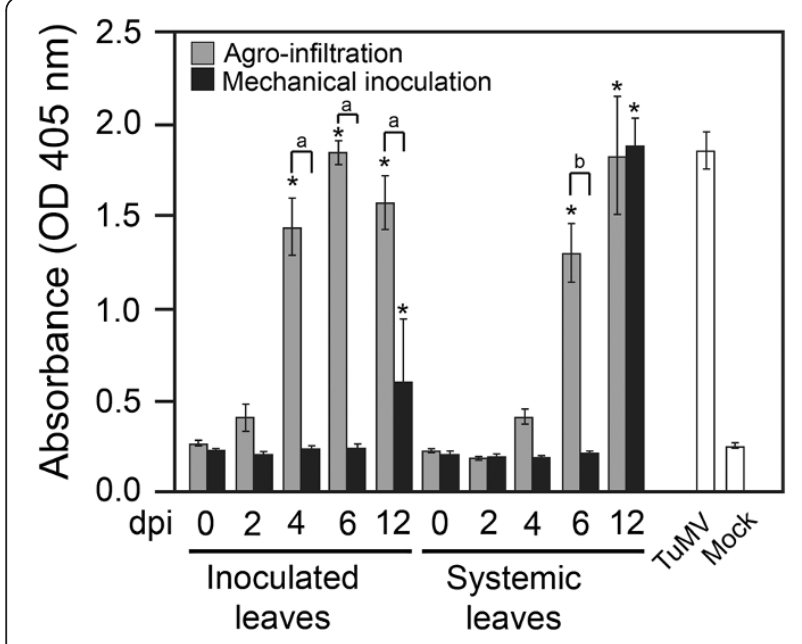

Figure 5 Comparison of TuMV initial infection efficiency between the agro-infiltration (pBD-TuMV-YC5) and mechanical inoculation (virus culture of TuMV YC5). Virus accumulated in vivo on inoculated or systemic leaves of $N$. benthamiana plants in which infection was initiated by either agro-infiltration or mechanical inoculation with TuMV infectious clones. The numbers indicated the days after inoculation (dpi). The virus titer was detected by ELISA with TuMV CP antiserum. The 12 dpi of TuMV-infected N. benthamiana plants (TuMV) were used as a positive control, whereas the mockinoculated plants (Mock) were the negative control. The samples of 2, 4,6 , and 12 dpi significantly different with the sample of 0 dpi in the same inoculation method and leaves position ( $p$-value $<0.05$ ) are indicated as "*". Significant difference between the agro-infiltration and the mechanical inoculation of the inoculated leaves under the same dpi ( $p$-value $<0.05)$ is indicated as "a". Significant difference between the agro-infiltration and the mechanical inoculation of the systemic leaves under the same dpi ( $p$-value $<0.05)$ is indicated as " $b$ ". Bars represent standard deviations $(n=3)$.

TuMV series caused systemic infection on $N$. benthamiana by agro-infiltration, which indicated that the pBD-TuMV series can be transferred through an agrobacterium transformation mechanism. In addition, agrobacteriummediated inoculation overcame the low infectivity rate when transferring the inocula prepared from $C$. quinoa plants to $N$. benthamiana plants. The reason for the low infectivity might due to the presence of components from C. quinoa plants that affect TuMV infection in $N$. benthamiana plants. However, this phenomenon did not show on ZYMV infection (Lin et al., 2007), suggesting that various viruses might have varying susceptibility to the components of $C$. quinoa during infection.

Virus accumulation was detected after $10 \mathrm{dpi}$ in plants inoculated by the virus culture of TuMV CY5, whereas by agro-infiltration, pBD-TuMV-CY5 caused viral accumulation on inoculated leaves at 4 dpi (Figure 5). Our explanation for this phenomenon is that agrobacterium was highly effective in transferring $\mathrm{T}$ DNA of the infectious clone into the nucleus, and these T-DNAs constitutively expressed TuMV viral RNA. We cannot exclude the possibility of synergistic symptoms caused by agrobacterium on primary infiltration of the $N$. benthamiana plants. However, this concern can be eliminated by the secondary transfer of TuMV from the primary infiltrated $N$. benthamiana plants to new plants. The secondarily inoculated plant will not have the interfering from agrobacterium. Therefore, the efficiency of transient expression of agro-infiltration plays an important role on initial infectivity. The 53\% initial infectivity of pBD-TuMV-GK might be caused by the variation in efficiency of the transient expression of agro-infiltration or might be because of the less infectious nature of the clone (Table 1).

Because of the highly effective transient expression of pBD003, the initial infectivity rate also increased in the TuMV-GK mild strain through pBD003-delivered inoculation (Table 1). The pBD003-delivered TuMV-GK demonstrated 53.3\% initial infectivity, and also shortened the process of infectivity, compared with the regular inoculation process using the p35S-TuMV infectious clone. The HC-Pro mutant $\left(\mathrm{R}_{182} \mathrm{~K}\right)$ was responsible for the low efficiency of the mild strain's initial infectivity, which consequently affected viral replication and resulted in low amounts of viral genome RNA. The TuMV-GK and ZYMV mild strain (ZYMV GAC) have a common $\mathrm{R}_{180}$ mutation on HC-Pro, which showed mild symptoms and a green fluorescence ring on C. quinoa (Figure 3A) (Lin et al., 2007). The results indicating the highly conserved $\mathrm{R}_{180}$ of HC-Pro of the potyvirus is a critical amino acid for symptoms development and local lesion formation of $C$. quinoa. Our previously study also showed that $\mathrm{R}_{180}$ of HC-Pro also plays an important role on suppression of the microRNA-mediated gene silencing pathway (Wu et al., 2010). In addition, the TuMV-GK also provides a good cross-protection on $N$. benthamiana to against the wild type TuMV (data not shown), indicating that the TuMV-GK can be used as a model for studying the mechanism of cross-protection in the future.

\section{Conclusion}

The newly constructed mini binary vector pBD003 provides a useful tool for research into host-virus interactions, viral infectious clone infectivity construction, and transient expression. The backbone of pBD003 contains no commonly used restriction enzyme sites, and the miniature size of the vector is advantageous for cloning. In this study, we provided a high initial infectivity of viral vector to study the virology and the functional genome of the virus. It can also be used to screen more TuMV mild strains that have various mutations on the viral genome for the cross-protection. Further investigation of the pBD003 mini binary vector can also be used on transient 
expression for gene expression and cell localization through agrobacterium transformation.

\section{Competing interests}

The authors declare that they have no competing interests.

\section{Authors' contributions}

S-SL first conceived the notion to design the pBD003 mini binary vector and expression TuMV in vivo infectious clone. Y-YL and M-MF performed the experiments. P-CL constructed pBD-TuMV-GK and M-TC produced TuMV CP antisera. L-YL performed the statistical analysis. C-PL provides specific comments for this study. All authors discussed the results and commented on the manuscript, which was written by Y-YL and S-SL. All authors read and approved the final manuscript.

\section{Acknowledgements}

We thank Dr. Savithramma Dinesh-Kumar for pTRV2 plasmid. This study was supported by a grant from the National Science Council (NSC 97-2321-B-002-046; NSC-98-2313-B-002-047-MY3; NSC 100-2923-B-002-001-MY3) to S.-S.Lin.

\section{Author details}

'Institute of Biotechnology, College of Bioresources and Agriculture, National Taiwan University, 81, Chang-Xing ST., Taipei 106, Taiwan. ${ }^{2}$ Genome and Systems Biology Degree Program, National Taiwan University, 1, Sec. 4, Roosevelt Rd., Taipei 106, Taiwan. ${ }^{3}$ Agriculture Biotechnology Research Center, Academia Sinica, 128, Sec. 2, Academia Rd., Taipei 115, Taiwan. ${ }^{4}$ Department of Plant Pathology and Microbiology, College of Bioresources and Agriculture, National Taiwan University, 1, Sec. 4, Roosevelt Rd., Taipei 106, Taiwan. ${ }^{5}$ Department of Agronomy, National Taiwan University, 1, Sec. 4, Roosevelt Rd., Taipei 106, Taiwan.

Received: 25 June 2012 Accepted: 5 December 2012

Published: 28 August 2013

\section{References}

Angenent GC, Posthumus E, Bol JF (1989) Biological activity of transcripts synthesized in vitro from full-length and mutated DNA copies of Tobacco rattle virus RNA 2. Virology 173:68-76

Boyer JC, Haenni AL (1994) Infectious transcripts and CDNA clones of RNA viruses. Virology 198:415-426

Cui X, Li G, Wang D, Hu D, Zhou X (2005) A Begomovirus DNAbeta-encoded protein binds DNA, functions as a suppressor of RNA silencing, and targets the cell nucleus. J Virol 79:10764-10775

Heaton LA, Carrington JC, Morris TJ (1989) Turnip crinkle virus infection from RNA synthesized in vitro. Virology 170:214-218

Hellens RP, Edwards EA, Leyland NR, Bean S, Mullineaux PM (2000) pGreen: a versatile and flexible binary Ti vector for Agrobacterium-mediated plant transformation. Plant Mol Biol 42:819-832

Huang Q, Hartung JS (2001) Cloning and sequence analysis of an infectious clone of Citrus yellow mosaic virus that can infect sweet orange via Agrobacterium-mediated inoculation. J Gen Virol 82:2549-2558

Janda M, French R, Ahlquist P (1987) High efficiency T7 polymerase synthesis of infectious RNA from cloned Brome mosaic virus CDNA and effects of $5^{\prime}$ extensions on transcript infectivity. Virology 158:259-262

Joh LD, VanderGheynst JS (2006) Agroinfiltration of plant tissues for production of high-value recombinant proteins: an alternative to production in transgenic crops. J Sci Food Agric 86:2002-2004

Kobayashi T, Antar AA, Boehme KW, Danthi P, Eby EA, Guglielmi KM Holm GH, Johnson EM, Maginnis MS, Naik S, Skelton WB, Wetzel JD, Wilson GJ, Chappell JD, Dermody TS (2007) A plasmid-based reverse genetics system for animal double-stranded RNA viruses. Cell Host and Microbe 1:147-157

Lin SS, Hou RF, Yeh SD (2002) Contruction of in vitro and in vivo infectious transcripts of a Taiwan strain of Zucchini yellow mosaic virus. Botanical Bull Acad Sinica 43:261-269

Lin SS, Wu HW, Jan FJ, Hou RF, Yeh SD (2007) Modifications of the helper component-protease of Zucchini yellow mosaic virus for generation of attenuated mutants for cross protection against severe infection. Phytopathology 97:287-296
Lin SS, Wu HW, Elena SF, Chen KC, Niu QW, Yeh SD, Chen CC, Chua NH (2009) Molecular evolution of a viral non-coding sequence under the selective pressure of amiRNA-mediated silencing. PLoS Pathog 5:e1000312

Liu Y, Schiff M, Marathe R, Dinesh-Kumar SP (2002) Tobacco Rar1, EDS1 and NPRI/NIM1 like genes are required for N-mediated resistance to Tobacco mosaic virus. Plant Journal 30:415-429

Llave C, Kasschau KD, Carrington JC (2000) Virus-encoded suppressor of posttranscriptional gene silencing targets a maintenance step in the silencing pathway. Proc Natl Acad Sci U S A 97:13401-13406

Maiss E, Timpe U, Brisske-Rode A, Lesemann DE, Casper R (1992) Infectious in vivo transcripts of a plum pox potyvirus full-length cDNA clone containing the cauliflower mosaic virus 35S RNA promoter. J Gen Virol 73:709-713

Melton DA, Krieg PA, Rebagliati MR, Maniatis T, Zinn K, Green MR (1984) Effcient in vitro synthesis of biologicafly active RNA and RNA hybridization probes from plsmids containing a bacteriophage SP6 promoter. Nucleic Acids Res 12:7035-7056

Niu QW, Lin SS, Reyes JL, Chen KC, Wu HW, Yeh SD, Chua NH (2006) Expression of artificial microRNAs in transgenic Arabidopsis thaliana confers virus resistance. Nat Biotechnol 24:1420-1428

Schnell MJ, Mebatsion T, Conzelmann KK (1994) Infectious rabies viruses from cloned CDNA. EMBO J 13:4195-4203

Shi BJ, Ding SW, Symons RH (1997) Plasmid vector for cloning infectious CDNAs from plant RNA viruses: high infectivity of CDNA clones of tomato aspermy cucumovirus. J Gen Virol 78:1181-1185

Wu HW, Lin SS, Chen KC, Yeh SD, Chua NH (2010) Discriminating mutations of HC-Pro of Zucchini yellow mosaic virus with differential effects on small RNA pathways involved in viral pathogenicity and symptom development. Mol Plant Microbe Interactions 23:17-28

doi:10.1186/1999-3110-54-22

Cite this article as: Lin et al:: Improving initial infectivity of the Turnip mosaic virus (TuMV) infectious clone by an mini binary vector via agroinfiltration. Botanical Studies 2013 54:22.

\section{Submit your manuscript to a SpringerOpen ${ }^{\circ}$ journal and benefit from:}

- Convenient online submission

Rigorous peer review

- Immediate publication on acceptance

- Open access: articles freely available online

- High visibility within the field

- Retaining the copyright to your article

Submit your next manuscript at $\gg$ springeropen.com 